\title{
tRNA-Derived Fragments as Novel Predictive Biomarkers for Trastuzumab- Resistant Breast Cancer
}

\author{
Chunxiao Sun ${ }^{a, b}$ Fan Yang ${ }^{a, b}$ Yanhong Zhang ${ }^{a, b} \quad$ Jiahui Chu ${ }^{a, b} \quad$ Jian Wang ${ }^{a, b}$ \\ Yifan Wang ${ }^{a, b}$ Yanqiu Zhang ${ }^{a, b}$ Jun Lia,b Yongfei Li ${ }^{a, b}$ Ruihua Fan ${ }^{a, d}$ Wei Li ${ }^{a}$ \\ Xiang Huang ${ }^{\mathrm{a}} \mathrm{Hao} \mathrm{Wu}^{\mathrm{a}}$ Ziyi Fu ${ }^{\mathrm{a}, \mathrm{c}}$ Zefei Jiang ${ }^{\mathrm{e}}$ Yongmei Yin ${ }^{\mathrm{a}}{ }^{\mathrm{f}}$
}

aDepartment of Oncology, the First Affiliated Hospital of Nanjing Medical University, Nanjing, ${ }^{\text {bThe }}$ First Clinical College of Nanjing Medical University, Nanjing, 'Nanjing Maternal and Child Health Medical Institute, Obstetrics and Gynecology Hospital Affiliated to Nanjing Medical University, Nanjing, dDepartment of Medical Oncology, Huai'an First People's Hospital, Nanjing Medical University, Huaian,

e Department of Breast Cancer, The 307 Hospital of Chinese People's Liberation Army, Beijing, ${ }^{\mathrm{f} J i a n g s u}$ Key Lab of Cancer Biomarkers, Prevention and Treatment, Collaborative Innovation Center for Cancer Medicine, Nanjing Medical University, Nanjing, China

\section{Key Words}

Breast cancer $•$ Trastuzumab resistance $\bullet$ tRNA-derived fragments

\begin{abstract}
Background/Aims: Resistance to trastuzumab remains a common challenge to HER-2 positive breast cancer. Up until now, the underlying mechanism of trastuzumab resistance is still unclear. tRNA-derived small non-coding RNAs, a new class of small non-coding RNA (sncRNAs), have been observed to play an important role in cancer progression. However, the relationship between tRNA-derived fragments and trastuzumab resistance is still unknown. Methods: We detected the levels of tRNA-derived fragments expression in normal breast epithelial cell lines, trastuzumab-sensitive and -resistant breast cancer cell lines using highthroughput sequencing. qRT-PCR was conducted to validate the differentially expressed fragments in serums from trastuzumab-sensitive and -resistant patients. A receiver operating characteristic (ROC) curve analysis was performed to evaluate the power of specific tRNAderived fragments. Progression-free survival (PFS) was analyzed using Cox-regression. Results: Our sequence results showed that tRNA-derived fragments were differentially expressed in the HBL-100, SKBR3, and JIMT-1 cell lines. tRF-30-JZOYJE22RR33 and tRF-27-ZDXPHO53KSN were found significantly upregulated in trastuzumab-resistant patients compared to sensitive individuals, and the ROC analysis showed that tRF-30-JZOYJE22RR33 and RRF-27ZDXPHO53KSN were correlated with trastuzumab resistance. In a multivariate analysis, higher levels of tRF-30-JZOYJE22RR33 and tRF-27-ZDXPHO53KSN expression were associated with significantly shorter PFS in patients with metastatic HER-2 positive breast cancer. Conclusion: Our results suggest that tRF-30-JZOYJE22RR33 and tRF-27-ZDXPHO53KSN play important

C. Sun, F. Yang and Y. Zhang contributed to this work equally.


roles in trastuzumab resistance. Patients with high levels of tRF-30-JZOYJE22RR33 and tRF-27ZDXPHO53KSN expression benefitted less from trastuzumab-based therapy than those that express lower-levels of these molecules. tRF-30-JZOYJE22RR33 and tRF-27-ZDXPHO53KSN may be potential biomarkers and intervention targets in the clinical treatment of trastuzumabresistant breast cancer.

\section{Introduction}

Human epidermal growth factor receptor-2 (HER-2) is overexpressed in $20-25 \%$ of human breast cancers [1]. Amplification of HER-2 confers a more aggressive phenotype and is associated with poor prognosis [2, 3]. Trastuzumab, a monoclonal anti-HER-2 antibody, has been shown to significantly improve clinical outcomes in the management of early and advanced breast cancers [4-6]. Despite this effectiveness, the disease will eventually progress in most patients who have received trastuzumab because of the development of primary or acquired resistance.

Up until now, trastuzumab resistance mechanisms have been found to mainly include the dysregulation of the downstream PI3K-AKT-mTOR pathway or loss of PTEN , accumulation of truncated kinase active p95-HER-2, overexpression of MUC4 , co-expression of other HER receptors such as HER3 or epidermal growth factor receptor (EGFR), overactivation of other signaling pathways such as insulin-like growth factor receptor signaling , among other potential mechanisms [7-10]. Although several treatment options, including trastuzumab emtansine (T-DM1), lapatinib, and continued trastuzumab in combination with pertuzumab have been applied clinically to overcome trastuzumab resistance [11-14], the benefit to patients has not been found significant [9]. Critically, the mechanism underlying trastuzumab resistance remains unclear, and there are currently no definite biomarkers to predict patients' responses to trastuzumab.

Transfer RNAs (tRNAs) are a fundamental component of the translation machinery. Several studies have indicated that tRNAs play roles in modulating cell proliferation and cancer progression [15-17]. tRNA halves (tRHs) and tRNA-derived small RNA fragments (tRFs) belong to a novel class of sncRNAs that are generated through precise cleavage of tRNAs [18-20]. tRNA halves are produced through specific cleavage in the anticodon loop under various stress conditions and are classified as 5'-tRNA halves (5'- tRHs) and 3'-tRNA halves (3'- tRHs) $[18,19]$. tRFs are generated through precise processing of both mature and precursor tRNAs (pre-tRNAs) and classified into at least three types, including the 5'- tRF, 3' - tRF, tRF-1 series. 5'- tRFs and 3' - tRFs are derived from the $5^{\prime}$-and $3^{\prime}$ - ends of mature tRNAs, respectively, whereas tRF-1s is derived from the $3^{\prime}$ ends of pre-mature tRNAs [20]. Another novel class of tRFs, which were identified recently as internal tRFs (i-tRFs), are typically derived from the internal region of mature tRNAs [21,22]. i-tRFs have atypical lengths, and there is no uniform standard to identify the length of these novel tRFs [21].

Recently, accumulated evidence has demonstrated that these tRNA-derived fragments play important roles in human cancers [23-29]. Lee YS et al. showed that knockdown of tRF-1001 significantly inhibit cell proliferation with specific accumulation of cells in the G2 phase and inhibition of DNA synthesis in prostate cancer [20]. i-tRFs and their mimics can suppress the stability of multiple oncogenic transcripts in breast cancer cells by displacing their 3' untranslated regions (UTRs) from the RNA-binding protein YBX1. Consequently, tRFs antagonize the activity of YBX1 and suppress breast cancer progression [23]. Additionally, tRNA halves can prevent apoptosis of cancer cells by binding to cytochrome $\mathrm{C}$ or promote the assembly of stress granules that help cells survive in adverse conditions [30]. Another recent study indicated that two specific tRFs derived from tRNA ${ }^{\text {Lys-CTT }}$ and tRNA ${ }^{\text {Phe-GAA }}$ were good indicators of progression-free survival (PFS) and candidate prognostic markers [31]. Taken together, these findings strongly suggest a functional role for tRNA-derived fragments in cancer progression. However, whether tRNA-derived fragments play a role in trastuzumabresistance is not well understood.

\section{KARGER}




\section{Cellular Physiology Cell Physiol Biochem 2018;49:419-431 \\ \begin{tabular}{l|l|l} 
and Biochemistry & $\begin{array}{l}\text { DOI: 10.1159/000492977 } \\
\text { Published online: 29 August } 2018\end{array}$ & $\begin{array}{l}\text { C) } 2018 \text { The Author(s). Published by S. Karger AG, Basel } \\
\text { www.karger.com/cpb }\end{array}$ \\
\hline
\end{tabular}}

Sun et al.: tRNA-Derived Fragments as Novel Predictive Biomarkers for TrastuzumabResistant Breast Cancer

In this study, we aimed to excavate trastuzumab-resistance related tRNA-derived fragments in HER-2 positive breast cancers, which might be helpful to understand the undergoing mechanisms of trastuzumab-resistance, and probably afford the potential biomarkers for diagnosis or therapy targets for clinical treatment.

\section{Materials and Methods}

\section{Patient samples and processing}

The study was approved by the Ethics Committee of the First Affiliated Hospital of Nanjing Medical University. Blood samples were collected from 57 women with HER-2 positive breast cancer after obtaining informed consent. The histopathological examination and immunohistochemistry of the primary and metastatic tissues were confirmed by two pathologists. The specimens with $2+$ immunostaining scores were identified as HER2 positive if the HER-2:CEP17 ratio was greater than or equal to 2 through fluorescence in situ hybridization (FISH). All of the patients were treated with trastuzumab during the adjuvant and/or advanced treatment. Whole blood samples were collected in EDTA-containing lavender top tubes (Becton Dickinson, NJ) before trastuzumab and chemotherapy treatments. Then, the samples were incubated at room temperature for 15 minutes and centrifuged at 5, $000 \times \mathrm{g}$ for 10 minutes. To remove the residual cells and debris, the serum supernatant was transferred to new tubes and centrifuged at 16,000 $\mathrm{g}$ for 15 minutes. Finally, the separated serum were stored at $-80^{\circ} \mathrm{C}$ until they were assessed. We used the unifying definition of trastuzumab primary resistance: progression at first radiological reassessment at 8-12 weeks or within 3 months after first-line trastuzumab in the metastatic setting or new recurrences diagnosed during or within 12 months after adjuvant trastuzumab [10]. There were 28 trastuzumab-sensitive patients and 29 patients with trastuzumab primary resistance in our data. The clinical characteristics of the patients are listed in Supplementary Table 1 (For all supplemental material see www.karger.com/doi/10.1159/000492977).

\section{Cell lines and culture conditions}

Trastuzumab-sensitive cell lines SKBR3 and trastuzumab primary resistant cell lines JIMT-1 were obtained from the American Type Culture Collection (ATCC, Manassas, VA, USA) [32, 33]. The normal breast epithelial cells lines HBL-100 was obtained from Cell bank of Chinese Academy of Sciences (Shanghai, China). SKBR3 cells were cultured in RPMI-1640 (Multicell, USA), and JIMT-1 along with HBL-100 cells were cultured in DMEM (Gibco, UK) with $10 \%$ fetal bovine serum (FBS, Gibco), containing $100 \mathrm{U} / \mathrm{ml}$ penicillin and $100 \mu \mathrm{g} / \mathrm{ml}$ streptomycin(Multicell, USA). Cells were cultured at $37^{\circ} \mathrm{C}$ in $5 \% \mathrm{CO}_{2}$, and the cell medium was replaced every $48 \mathrm{~h}$.

\section{Cell Viability assay}

SKBR3 and JIMT-1 cells (3000/well) were planted in 96-well plates. After 24 hours, they were treated with trastuzumab $(0,0.2,2,20,200 \mu \mathrm{g} / \mathrm{ml}, 2 \mathrm{mg} / \mathrm{ml})$ for $1,2,3,4$, or 5 days. Cell viability studies was analyzed using a Cell Counting Kit-8 (CCK8, Dojindo) according to the manufacturer's instructions [34]. The optical density values were measured at $450 \mathrm{~nm}$.

\section{Flow cytometry analysis}

SKBR3 and JIMT-1 cells were harvested after $20 \mu \mathrm{g} / \mathrm{ml}$ trastuzumab for 48 and 72 hours. After incubation with Annexin V-FITC and propidium iodide (PI) (BD Bioscience, San Jose, CA) according to the manufacturer's recommendations [35], cell apoptosis was then analyzed using flow cytometry (FACScan; BD Biosciences) equipped with a Cell Quest software (BD Biosciences).

\section{RNA isolation}

Total RNA was extracted from cells using Trizol reagent (Life Technologies, USA) according to the manufacturer's protocol, and the RNA was prepared for high-throughput sequencing. Total RNA from patients' serums were isolated with Trizol LS (Life Technologies, USA), following the manufacturer's protocol. The quality and concentration of the isolated RNA were assessed using an Agilent 2100 Bioanalyzer (Thermo Scientific). The OD 260/280 absorbance ratios of all of the samples were between 1.8 and 2.0. Final RNA preparations were resuspended in RNase-free water and stored at $-80^{\circ} \mathrm{C}$.

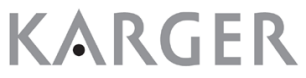




\section{RNA sequence processing and expression analysis}

Illumina NextSeq 500 raw sequencing reads that passed the Illumina chastity filter were used for the sequence analysis. After quality control, the sequencing reads were 5', 3'-adaptor trimmed, filtered for $\geq 16$ nt using cutadapt software. Then the sequencing reads were aligned to mature-tRNA on the entire genome using MINTmap (https://github.com/TJU-CMC-Org/MINTmap/) [36]. Briefly, genomic tRNA sequences are processed to simulate exon splicing, and then get modified to admit the non-templated CCA additi and the "- 1" nucleotide of tRNA. The resulting sequences are fragmented computationally into (overlapping) segments of variable lengths and entered into a lookup table: sequences that are not exclusive to tRNA space are flagged at this point using metadata added to the table. The lookup table is then used to process a (quality-filtered and adaptertrimmed) short RNA-seq dataset to generate a tRF expression profile table. For each tRF, MINTmap identified whether it is exclusive to tRNA space [37, 38]. Because tRF-1s are derived from the precursor tRNAs, they require a different type of analytical work than fragments that overlap with the mature tRNA, and as such, tRF-1s were not included in our data. The adjusted p-values lower than 0.05 were considered significant. The data for high-throughput sequencing are deposited at GEO under the accession number GSE107473.

\section{Quantitative real-time PCR}

Total RNA extracted from cells and clinical samples were pretreated with an rtStar ${ }^{\mathrm{TM}}$ tRF\&tiRNA Pretreatment Kit (Arraystar, USA) to remove terminal and internal methylations for efficient qRT-PCR. Then, the RNA was quantified by qRT-PCR using a Bulge-Loop miRNA qRT-PCR Stater Kit (Ribobio, China) according to the manufacturer's instructions [39]. Briefly, the post-treated RNA was reverse transcribed to cDNA using stem loop primers specific for each target tRNA-derived fragments of interest. The RT mixtures were incubated for $60 \mathrm{~min}$ at $42^{\circ} \mathrm{C}$ and $10 \mathrm{~min}$ at $70^{\circ} \mathrm{C}$. Then, qPCR was performed using SYBR Green Mix containing Taq enzyme, dNTP mix, PCR buffer, and SYBR Green I [39]. After adding forward primer and reverse primers, the reaction mixtures were incubated at $95^{\circ} \mathrm{C}$ for $10 \mathrm{~min}$, followed by 40 cycles of $95^{\circ} \mathrm{C}$ for $2 \mathrm{~s}, 60^{\circ} \mathrm{C}$ for $20 \mathrm{~s}$, and $70^{\circ} \mathrm{C}$ for $10 \mathrm{~s}$ in an Applied Biosystems 7300 Real Time PCR System (Applied Biosystems, Foster City, CA). U6 were used as an internal control [21, 40]. The $2^{-\Delta \Delta C t}$ and $\Delta \mathrm{Ct}[34,41]$ methods were used for analysis of the differential expression of tRFs in cells and serum samples, respectively. The names of tRFs, their sequences and specific primer sequence are given in Supplementary Table 2.

\section{Statistical analysis}

The differences in tRF levels were determined with an ANOVA and multiple hypothesis testing. The optimal sensitivity and specificity determined from ROC curves were used in the standard method. An FDR value or p-value less than 0.05 was considered statistically significant. Calculations were conducted using SPSS version 20.0 (SPSS Inc., Chicago, USA) and GraphPad Prism 6 (GraphPad Software, California, USA).

\section{Results}

Effect of trastuzumab on the viability of SKBR3 and JIMT-1 cells

In order to confirm the sensitivity of SKBR3 and JIMT-1 cells to trastuzumab, CCK-8 and flow cytometry analysis were carried out to determine the cell proliferation and apoptosis rates after trastuzumab treatment. Cell proliferation was confirmed by constructing timedependent and concentration-dependent growth curves. Inhibition of cell viability increased in both SKBR3 and JIMT-1 cells with an increase in the concentration of trastuzumab, whereas inhibition of SKBR3 was greater than that in JIMT-1 cells (Fig. 1A). In addition, the loss of SKBR3 cell viability was $>30 \%$ compared to that of JIMT- 1 cells after 5 days of $20 \mu \mathrm{g} /$ ml trastuzumab (Fig. 1B), which was consistent with a previous study [33]. Furthermore, apoptosis of SKBR-3 cells was significantly increased compared to that of JIMT-1 cells after treatment with $20 \mu \mathrm{g} / \mathrm{ml}$ trastuzumab (Fig. $1 \mathrm{C}$ and D).These results indicated that SKBR3 cells were sensitive to trastuzumab, whereas JIMT-1 cells were resistant to trastuzumab. 
tRNA-derived fragments expressed in HBL-100, SKBR3, and JIMT-1 cells

We used a high-throughput sequencing technique to determine differential expression of tRNA-derived fragments in normal breast epithelial cell lines, trastuzumab-sensitive and -resistant breast cancer cell lines. The copy number and sequences of each unique read in the distribution of sequence read lengths were recorded (Supplementary Fig. 1A-C). Based on the results of sequencing analysis, 6309 tRNA-derived fragments were identified in the HBL-100, SKBR3, and JIMT-1 cell lines. All of the cytosolic tRNAs and 18 mitochondrial

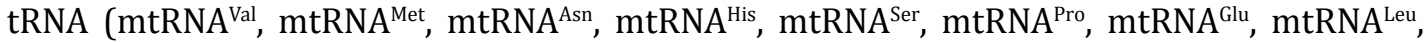

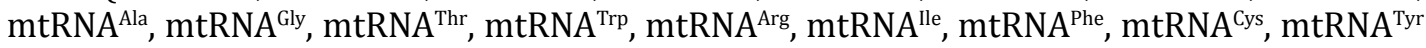
, mtRNA $^{\text {Asp }}$ ) were found to produce tRNA-derived fragments. After further screening, a total of 998 tRNA-derived fragments were identified with fold-change filtering (absolute foldchange $>2.0$ ), a standard Student's t-test $(\mathrm{p}<0.05)$, and multiple hypothesis testing (FDR < 0.05 ). In addition, tRNAs with different sequences may have the same anticodon and transfer the same amino acid. We divided the tRNAs into several groups with the same anticodons. tRFs and tRNA halves derived from the same anticodon tRNA are showed in Supplementary Fig. 1D-F.

\section{Differentially expressed tRFs and tRNA halves in HER-2 positive and trastuzumab-resistant} cell lines

To analyze the tRNA-derived fragments differentially expressed in HBL-100, SKBR3 and JIMT-1 cells, all of the differentially expressed tRNA-derived fragments were compared in a heatmap (Fig. 2A). The differences among the three cell lines are shown by a principal component analysis (Supplementary Fig. 2). In addition, the percentage of each subtype of differentially expressed tRNA-derived fragments indicated that more than $80 \%$ of the fragments were tRFs in all three cell lines (Fig. 2B-D). The scatter plot indicated that the expression level in the two groups (up- and down-regulated fragments) in all three cell lines correlated well (Fig. 3A-C). All of the upregulated and downregulated tRNA-derived fragments are summarized in Fig. 3D (SKBR3 vs. HBL-100; JIMT-1 vs. HBL-100; JIMT-1 vs. SKBR3). Because SKBR3 and JIMT-1 are both HER-2 positive cell lines from different sources [32, 33], we first selected those fragments that were not only differentially expressed between SKBR3 and HBL-100 but also that were differentially expressed in JIMT-1 and HBL-100 cells. We found several fragments to be significantly differentially expressed in HER-2 positive tumor cell lines (SKBR3 and JIMT-1) when compared to the normal breast epithelial cell line (HBL100). In total, 36 upregulated and 21 downregulated tRNA-derived fragments with read

Fig. 1. Validation of the sensitivity of SKBR3 and JIMT-1 cell lines to trastuzumab. Cell viability in the presence of various concentrations of trastuzumab $(0,0.2,2,20$, $200 \mu \mathrm{g} / \mathrm{ml}, 2 \mathrm{mg} / \mathrm{ml}$ ) for 5 days determined by CCK8 assay (A). Cell viability curves for JIMT-1 and SKBR3 cells grown for 5 days in $20 \mu \mathrm{g} / \mathrm{ml}$ trastuzumab (B). The apoptosis rate in SKBR3 and JIMT1 cells was determined by flow cytometry analysis (C-D). Asterisks indicate significant differences between groups (“*”: $p<0.05$, “**”: $\mathrm{p}<0.01, \mathrm{n}=3$ ).

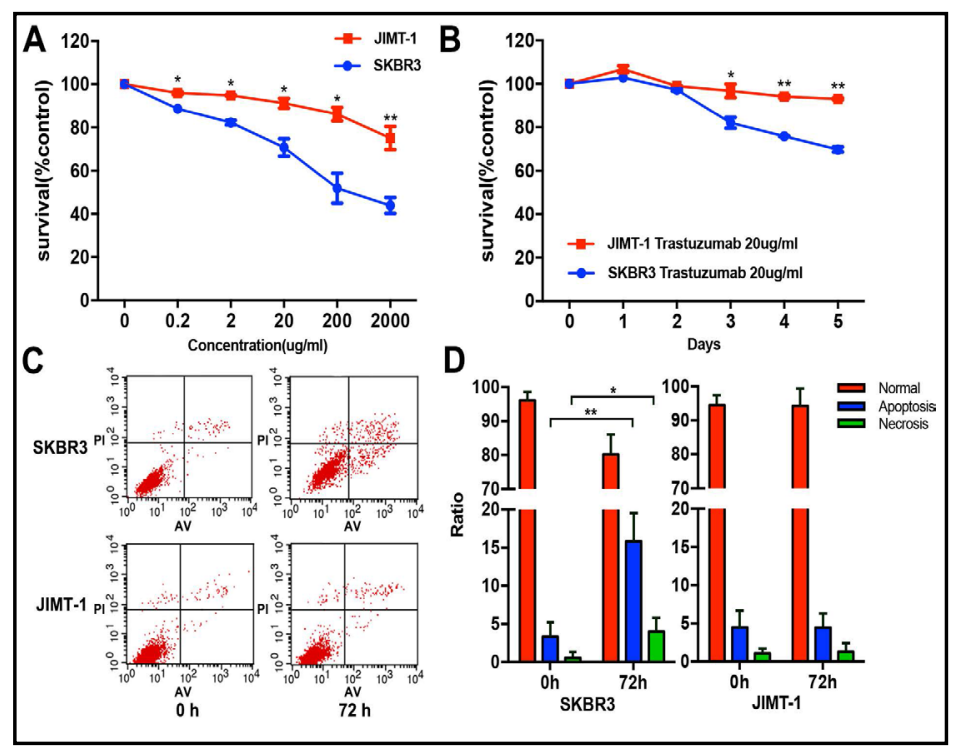


density more than 100 were identified as differentially expressed in SKBR3 and JIMT-1 cells compared to those in HBL-100 (Fig. 3E). Subsequently, we found 11 upregulated and 1 downregulated tRFs in JIMT-1 cells compared with those in SKBR3 cells, among the 57 dysregulated tRNA-derived fragments described above. Finally, we selected 5 upregulated (tRF-30-JZOYJE22RR33, tRF-27-ZDXPHO53KSN, tRF-26-XIP $2801 \mathrm{MK} 8 \mathrm{E}$, tRF-29-IYEVFMD 0SR1Z, tRF-22-8B8871K92) and 1 downregulated (tRF30-SERXPIN2NYDR) tRFs with the most significant difference for further investigation. According to the cleavage position on the cloverleaf secondary structure of the derived

Fig. 3. Differentially expressed tRFs and tRHs in HER-2 positive and trastuzumab-resistant cell lines. tRFs and tRHs expression levels were measured and normalized as tag counts per million of total aligned tRNA reads (TPM). The values of $X$ and $Y$ axes in the scatter-plot are the averaged TPM values of each group $\left(\log _{2}\right.$ scaled). Genes above the top line (red dots, up-regulation) or below the bottom line (green dots, downregulation) showed changes of more than 2.0 fold (default fold change value is 2.0 ) between the two comparison groups. Gray dots indicate tRFs and tRNHs that were not differentially expressed. (A) SKBR3 vs. HBL-100. (B) JIMT-1 vs. HBL-100. (C) JIMT-1 vs. SKBR3. (D) All of the upregulated and

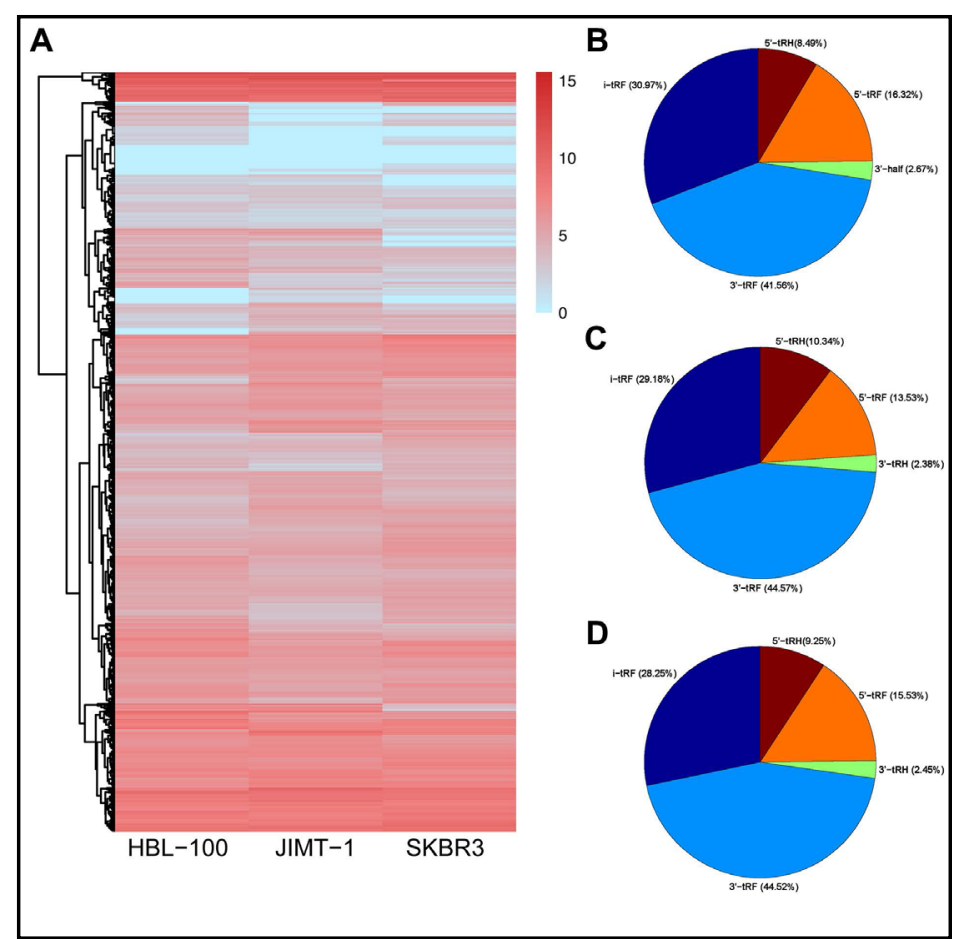

Fig. 2. tRNA-derived fragments expression profiling and subtypes in HBL-100, SKBR3, and JIMT-1 cells. Heatmaps of differentially expressed tRNA-derived fragments in HBL-100, SKBR3 and JIMT-1 cells (A). Differential expression of each subtype of unique tRNA-derived fragments in HBL-100 (B), SKBR3 (C), JIMT-1 (D) cells.

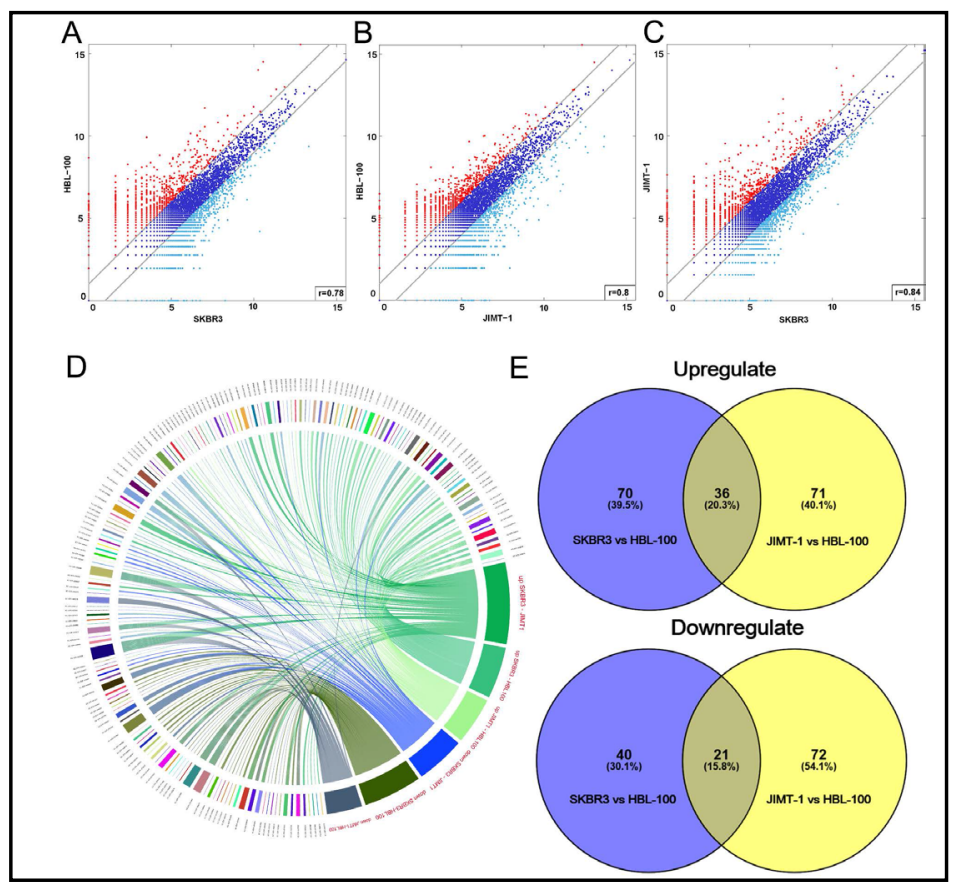
downregulated tRNA-derived fragments in each of the two groups are summarized in the circle. (E) Thirtysix upregulated and 21 downregulated tRNA-derived fragments were identified as differently altered in SKBR3 and JIMT-1 cells compared to those in with HBL-100 cells.

\section{KARGER}


tRNAs, all of these tRFs were identified as i-tRFs (Fig. 4A). Then, we verified the expression levels of the dysregulated tRFs in the HBL-100, SKBR3 and JIMT-1 cell lines by qRT-PCR. The qRT-PCR results showed that tRF-30-JZOYJE22RR33, tRF-27-ZDXPHO53KSN, and tRF-26-XIP2801MK8E were significantly upregulated and tRF-30-SERXPIN2NYDR was significantly downregulated in both two HER-2 positive cell lines (compared with HBL100) and in trastuzumab-resistant cells (compared with SKBR3) (Fig. 4B). The differential expression of tRF-29-IYEVFMD0SR1Z and tRF-22-8B8871K92 were not fully consistent with the expression trends in HER-2 positive cells and trastuzumab-resistant cells. These results indicated that several dysregulated tRFs may play important roles in primary resistance to trastuzumab in HER-2 positive breast cancer.

\section{Specific tRFs are associated with resistance to trastuzumab and poor prognosis}

In order to determine the association between the expression of dysregulated tRFs and patients' responses to trastuzumab, qRT-PCR was performed to quantify the expression of dysregulated tRFs in clinical serum samples. In total, the expression of these eight selected tRFs was validated in the sera of 28 trastuzumab sensitive patients and 29 patients with primary resistance. The results showed that tRF-30-JZOYJE22RR33 and tRF-27-ZDXPHO53KSN (both derived from tRNA ${ }^{\text {Cys-GCA }}$ ) were significantly upregulated in patients with primary resistance compared to those in trastuzumab-sensitive patients (Fig. 5A and B). We did not detect a statistically significant difference in the expression of tRF-26-XIP2801MK8E and tRF-30SERXPIN2NYDR (Fig. 5C and D). tRF-29-IYEVFMD0SR1Z and tRF-22-8B8871K92 were not detected in our serum samples. In addition, a ROC curve analysis was performed to evaluate the power of tRF-30-JZOYJE22RR33 and tRF-27-ZDXPHO53KSN to predict trastuzumab resistance. The results showed that the ROC curve for tRF-30-JZOYJE22RR33 had an area under the curve (AUC) of 0.7147 ( $\mathrm{P}$ value $=0.02370,95 \% \mathrm{CI}=0.5476$ to 0.8817 ) (Fig. $5 \mathrm{E}$ ), and the tRF-27-ZDXPH053KSN AUC was 0.8308 ( $\mathrm{P}$ value $=0.00298,95 \% \mathrm{CI}=0.6811$ to 0.9805 ) (Fig. 5F). In addition, four prognostic factors were identified in a univariate analysis: primary tumor stage (I/II, III/IV), Ki67 ( $\leqslant 30 \%,>30 \%)$, tRF-30-JZOYJE22RR33, and tRF-

Fig. 4. Validation of six dys-regulated tRNA-derived fragments associated with trastuzumab resistance in HBL-100, SKBR3, and JIMT-1 cells. Examples of tRNA or pre-tRNA structures for each species of the six identified tRNA-derived fragments were depicted. The black nucleotides at the 3' end indicate the presence of a terminal CCA sequences (A). qRT-PCR analysis of tRF-30-JZOYJE22RR33, tRF-27-ZDXPH053KSN, tRF-26-XIP2801MK8E, tRF-29-IYEVFMD0SR1Z, tRF-22-8B8871K92, and tRF-30-SERXPIN2NYDR expression in HBL-100, SKBR3 and JIMT-1 cells. Gene expression was normalized to U6 expression, and fold differences were calculated using the $2^{-\Delta \Delta \mathrm{ct}}$ method by comparing gene expression levels to those in HBL-100 cells (B). All data were analyzed using the Student's t test. Asterisks indicate significant difference between groups (“*”: $p<0.05$, “**”: $\mathrm{p}<0.01, \mathrm{n}=3$ ).

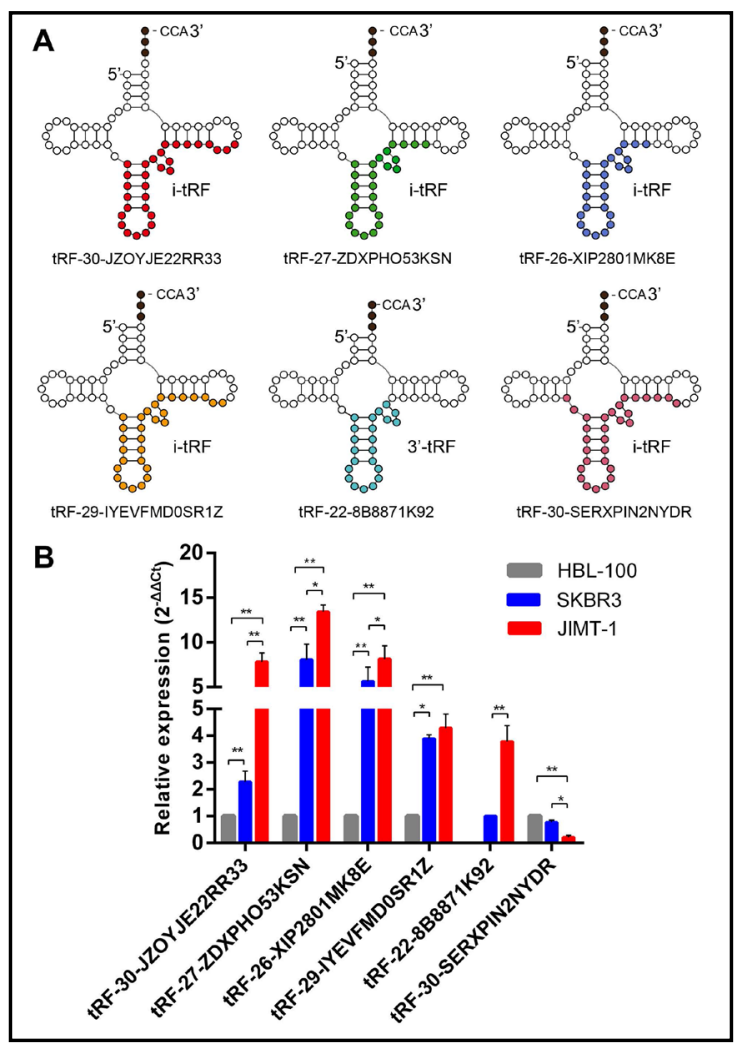


Fig. 5. Validation of tRF-30JZOYJE22RR33, tRF-27-ZDXPHO53KSN, tRF-26-XIP2801MK8E, tRF-29-IYEVFMD0SR1Z, tRF-228B8871K92, and tRF-30-SERXPIN2NYDR expression in the sera of trastuzumab-sensitive and -resistant patients. tRF-30JZOYJE22RR33 and tRF-27-ZDXPH053KSN were significantly upregulated in trastuzumab-resistant patients compared to trastuzumab-sensitive individuals (A and B). The difference in expression of tRF-26-XIP2801MK8E and tRF30-SERXPIN2NYDR between the sensitive and resistant groups was not statistically significant (C

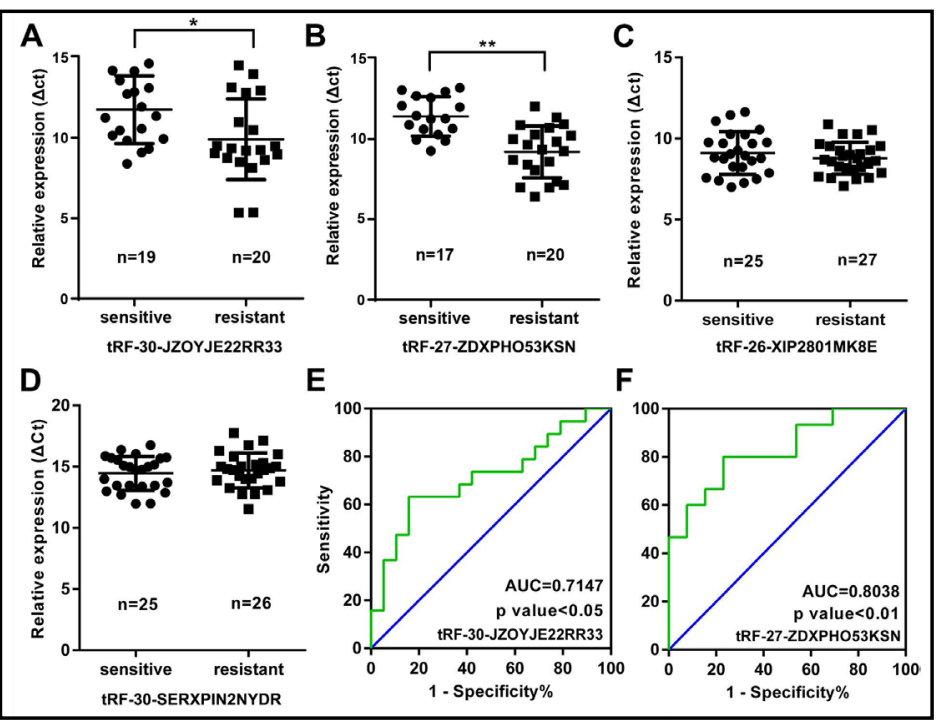
and D). tRF-29-IYEVFMD0SR1Z and tRF-22-8B8871K92 were not detected in serum samples. A ROC curve analysis showed the power of tRF-30-JZOYJE22RR33 and tRF-27-ZDXPHO53KSN to predict trastuzumab resistance ( $\mathrm{E}$ and $\mathrm{F}$ ).

Table 1. Univariate and multivariate analyses of the clinicopathological factors for PFS in 52 patients with metastatic breast cancer. Lower $\Delta$ CT represent high expression of tDR-1960 and tDR-1969. ( ${ }^{*} \mathrm{P}<0.05$. ${ }^{* *} \mathrm{P}<0.01$.Abbreviation: HR, hazard ratio)

\begin{tabular}{|c|c|c|c|c|}
\hline \multirow{2}{*}{ Risk factors } & \multirow{2}{*}{$\begin{array}{c}\text { Univariate analysis } \\
\text { P-Value }\end{array}$} & \multicolumn{3}{|c|}{ Multivariate analysis } \\
\hline & & HR & $\begin{array}{c}\text { P- } \\
\text { Value }\end{array}$ & $95 \% \mathrm{CI}$ \\
\hline tRF-30-JZOYJE22RR33 $(\Delta \mathrm{CT} \leqslant 11.0,>11.0)$ & $0.002^{* *}$ & 2.754 & $0.040^{*}$ & $1.038-5.219$ \\
\hline tRF-27-ZDXPHO53KSN $(\Delta \mathrm{CT} \leqslant 10.5,>10.5)$ & $0.004^{* *}$ & 2.265 & $0.019 *$ & $1.187-6.756$ \\
\hline Primary tumor stage(I/II, III/IV) & $0.011^{*}$ & 2.696 & $0.015^{*}$ & $1.197-5.243$ \\
\hline $\mathrm{Ki} 67 \%(\leqslant 30 \%,>30 \%)$ & $0.012^{*}$ & 1.461 & 0.081 & $0.913-4.744$ \\
\hline $\operatorname{Age}(\leqslant 50,>50)$ & 0.872 & & & \\
\hline Prior adjuvant Trastuzumab treatment & 0.343 & & & \\
\hline Prior endocrine therapy & 0.477 & & & \\
\hline Estrogen receptor (positive, negative) & 0.512 & & & \\
\hline Progesterone receptor (positive, negative) & 0.74 & & & \\
\hline Chemotherapy(taxanes, non taxanes) & 0.639 & & & \\
\hline Line of chemotherapy $(\leqslant 1,>1)$ & 0.76 & & & \\
\hline
\end{tabular}

27-ZDXPH053KSN expression (Table 1). High expression levels of tRF-30-JZOYJE22RR33 and tRF-27-ZDXPHO53KSN were associated with worse PFS (Fig. 6A and B). No relationship between PFS and other factors, for example, age $(\leq 50,>50)$, estrogen receptor (positive, negative), progesterone receptor (positive, negative), chemotherapy (taxanes, non taxanes), was found in our study. Notably, a multivariate analysis further revealed that higher expression of tRF-30-JZOYJE22RR33 and tRF-27-ZDXPH053KSN, as well as primary tumor stage $(P=0.015$; Table 1$)$, were associated with significantly shorter $P F S(P=0.040,0.019)$. Therefore, reasoned that patients with high levels of tRF-30-JZOYJE22RR33 and tRF-27ZDXPHO53KSN expression would benefit less from trastuzumab-based therapy. tRF-30JZOYJE22RR33 and tRF-27-ZDXPH053KSN could be potential biomarkers and intervention targets for the clinical treatment of trastuzumab-resistant breast cancer. 
Fig. 6. Progression-free survival (PFS) for the 52 metastatic HER-2 positive breast cancer patients who received advanced Trastuzumab therapy. (A). Patients with high level of tRF-30-JZOYJE22RR33 expression $(\Delta \mathrm{CT} \leqslant 11.0)$ had significantly shorter PFS $(\mathrm{P}=0.002)$ than patients with low level of tRF-30JZOYJE22RR33 expression ( $\Delta \mathrm{CT}>11.0)$. (B) Patients with high level of tRF-27-ZDXPH053KSN expression $(\Delta \mathrm{CT} \leqslant 10.5)$ had significantly shorter PFS $(\mathrm{P}=0.004)$

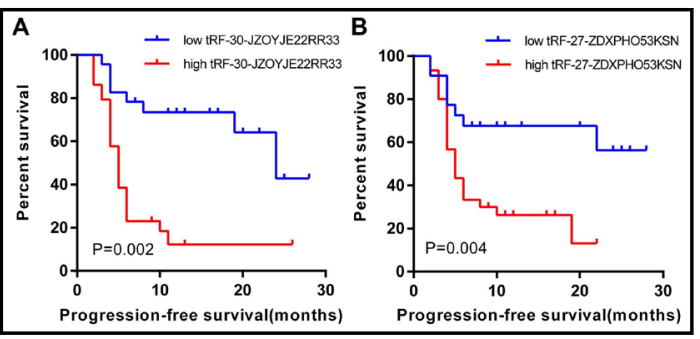
than those with low expression of tRF-27-ZDXPH053KSN ( $\Delta \mathrm{CT}>10.5$ ) (“*”: $p<0.05$, “**”: $\mathrm{p}<0.01, \mathrm{n}=3$ ).

\section{Discussion}

Despite numerous efforts to identify biomarkers of response to trastuzumab in HER-2 positive patients, there are currently no definitive biomarkers available for use in clinical practice. With the development of genome sequencing technologies, tRNA-derived fragments have been identified in association with a number of human diseases, including cancer, infection, neurodegeneration, and other pathological conditions. tRFs and tRNA halves with dysregulated expression in cancer were considered as potential diagnostic biomarkers or therapeutic targets in cancer cells [42].

To explore the potential role of tRNA-derived fragments in trastuzumab resistance, we performed high-throughput sequencing to determine the expression profile of tRFs and tRHs in normal breast epithelial cell lines and cells sensitive and resistant to trastuzumab. Based on the sequencing data, we conducted a comprehensive analysis of tRNA-derived fragments in HER-2 positive breast cancer cells in comparison with that in normal epithelial cells (Fig. 2A). The sequencing data showed that tRFs were more common than tRNA halves in all three cell lines (Fig. 2B-D). Shozo Honda et al. demonstrated that several tRNA halves were prominently enriched only in estrogen receptor (ER) positive breast cancer cell lines and patient tissues, whereas ER negative breast cancer cell lines and tissues expressed low levels of tRNA halves [40]. Because both SKBR3 and JIMT-1 both are ER negative cell lines, this may explain why the expression of tRNA halves were less than tRFs in our data structure. Therefore, we reason that sex hormone sensitivity and hormone receptor expression could be associated with the production of tRFs and tRNA haves in breast cancer.

Initially, tRFs were classified into tRF-5, tRF-3, and tRF-1 series found in prostate cancer [20]. Recently, Aristeidis et al. found a novel fragment class, referred to as i-tRFs, in breast cancer cell lines and tumor tissues [21]. Until now, there has been little information on the biological function of i-tRFs in cancer progression. In our sequencing data, we detected four series of tRFs and also found a number of i-tRFs in HER-2 positive breast cancer cells. i-tRFs are wholly internal to the respective mature tRNAs, and can straddle the anticodon. We also showed examples of tRNA structures for each species, depicting the positions of the identified tRFs (Fig. 4A). The composition and abundance in the transcriptome of tRNAderived fragments has been proposed to be dependent on gender, tissue, disease and even disease subtype [21]. On the other hand, we may reasonably infer that the expression of $\mathrm{i}$-tRFs in breast cancer cell lines, tissues and serums are common, and might be a general characteristic of the tRFs expression in breast cancer.

To select tRNA-derived fragments relevant to trastuzumab resistance, we chose fragments among those that were differentially expressed in SKBR3 and JIMT- 1 vs. HBL-100 cells to confirm the stable expression of fragments in HER-2 positive cell lines. Then, we used the sera of HER-2 positive patients treated with trastuzumab to validate the differential expression of selected tRFs in JIMT-1 and SKBR3. qRT-PCR was conducted to detect the differential expression in trastuzumab-sensitive and -resistant patients. Previous studies showed that tRF expression has been detected in cancer patient samples from multiple KARGER 
tissue sites and accessible samples like human urinary [43-45]. In the 1970s, Borek E found the presence of tRNA-derived fragments in the serums of cancer patients [46]. Recently, Dhahbi et al. reported that tRFs in serum were circulating at different levels in breast cancer patients compared to those in healthy individuals [47]. However, no further studies have explored the potential roles of these tRNA-derived fragments in the serum of cancer patients or their biological functions in the progression of the cancer [48]. Our data also elucidated the origin of tRNA-derived fragments in SKBR3, JIMT-1, and HBL-100 cells and analyzed the differential expression of these molecules (Fig. 2 and 3). In addition, tRF-30-JZOYJE22RR33 and tRF-27-ZDXPHO53KSN were found to be overexpressed in trastuzumab-resistant patients compared to drug sensitive individuals (Fig. 5A and B). The ROC analysis showed that tRF-30-JZOYJE22RR33 and tRF-27-ZDXPH053KSN were associated with trastuzumab resistance (Fig. 5E and F). Remarkably, overexpression of tRF-30-JZOYJE22RR33 and tRF-27ZDXPH053KSN predicted a poor prognosis and could be regarded as independent predictors of PFS in HER-2 positive breast cancers (Fig. 6A and B). The development of biomarkers is a complicated process. A convenient and high-efficiency method for patient sample collection and analysis of extracellular RNA will be critical as biomarker studies of tRFs move forward [49-51]. Given the broad range of human tumors in which tRNA-derived fragments are dysregulated, our observations in the serum of breast cancer patients resistant to trastuzumab may be more generally applicable to other malignant tumors and tRNA-derived fragments may be used as fluid-based biomarkers for future prospective screening studies.

Exploration of the mechanism of tRF/tRH-mediated cancer progression is still at its infancy. Different types of tRNA-derived fragments have been found to have a variety of different functions [29]. 5'-tRHs, but not 3'-tRHs, play a role in inhibiting protein synthesis and promoting the assembly of stress granules $[52,53]$. tRFs have been identified to copurify with Argonaute and Piwi complexes, which suggests that such tRNA-derived fragments could function as miRNAs or piRNAs [54-56]. Recently 5'-tRF series were also found to inhibit translation independently of siRNA [57]. Specific i-tRFs have been shown to compete with mRNAs for the binding to RNA-binding proteins in breast cancer [23]. In addition, tRF-1001 derived from a pre-tRNA is necessary for cell growth, and knockdown of tRF-1001 causes significant inhibition of cell proliferation in prostate cancer [20]. Our results indicate that i-tRFs, which have rarely been studied, are involved with trastuzumab resistance. On the other hand, some limitations of our study should also be acknowledged. Firstly, the verification method of Mircury/Taqman may measure similar molecules at the same time. In addition, we have no further research about the mechanism of tRFmediated trastuzumab resistance. However, based our previous bioinformatic analysis (data not present), we speculate that tRF-30-JZOYJE22RR33 and tRF-27-ZDXPHO53KSN may participate in trastuzumab resistance through regulating the expression product of target genes or competing with mRNAs for the binding to RNA-binding proteins. The mechanism underlying the effects of tRNA-derived fragments on trastuzumab resistance is extremely complicated and further research is needed.

In conclusion, we comprehensively analyzed tRNA-derived fragments in trastuzumabsensitive and -resistant breast cancer. We also identified tRF-30-JZOYJE22RR33 and tRF-27ZDXPHO53KSN as potential therapeutic response and prognostic biomarkers and as possible intervention targets for trastuzumab-resistant breast cancer. We believe that results of our study could provide leads for the further exploration of biological functions of these novel tRNA -derived fragments in trastuzumab-resistant breast cancer.

\section{Acknowledgements}

This study was financially supported by the National Natural Science Foundation of China $(81172503,81572603,81302304,81402139$, and 81570804), the Project of Jiangsu 


\section{Cellular Physiology Cell Physiol Biochem 2018;49:419-431 \\ \begin{tabular}{l|l|l} 
and Biochemistry & $\begin{array}{l}\text { DOI: 10.1159/000492977 } \\
\text { Published onlIne: 29 August } 2018\end{array}$ & $\begin{array}{l}\text { C) } 2018 \text { The Author(s). Published by S. Karger AG, Basel } \\
\text { www.karger.com/cpb }\end{array}$ \\
\hline
\end{tabular}}

Sun et al.: tRNA-Derived Fragments as Novel Predictive Biomarkers for TrastuzumabResistant Breast Cancer

Provincial Medical Talent (ZDRCA2016023), the Project of Wu Jieping Medical Foundation (320.6750.17006), and the Second level of Jiangsu "333" Personnel Training Project (BRA2017534).

\section{Disclosure Statement}

The authors declare that they have no conflict of interests.

\section{References}

-1 Slamon DJ, Clark GM, Wong SG, Levin WJ, Ullrich A, McGuire WL: Human breast cancer: Correlation of relapse and survival with amplification of the her-2/neu oncogene. Science 1987;235:177-182.

-2 Andrulis IL, Bull SB, Blackstein ME, Sutherland D, Mak C, Sidlofsky S, Pritzker KP, Hartwick RW, Hanna W, Lickley L, Wilkinson R, Qizilbash A, Ambus U, Lipa M, Weizel H, Katz A, Baida M, Mariz S, Stoik G, Dacamara P, et al.: Neu/erbb-2 amplification identifies a poor-prognosis group of women with node-negative breast cancer. Toronto breast cancer study group. J Clin Oncol 1998;16:1340-1349.

-3 Sjogren S, Inganas M, Lindgren A, Holmberg L, Bergh J: Prognostic and predictive value of c-erbb-2 overexpression in primary breast cancer, alone and in combination with other prognostic markers. J Clin Oncol 1998;16:462-469.

4 Slamon DJ, Leyland-Jones B, Shak S, Fuchs H, Paton V, Bajamonde A, Fleming T, Eiermann W, Wolter J, Pegram M, Baselga J, Norton L: Use of chemotherapy plus a monoclonal antibody against her2 for metastatic breast cancer that overexpresses her2. N Engl J Med 2001;344:783-792.

5 Dahabreh IJ, Linardou H, Siannis F, Fountzilas G, Murray S: Trastuzumab in the adjuvant treatment of earlystage breast cancer: A systematic review and meta-analysis of randomized controlled trials. Oncologist 2008;13:620-630.

6 Paik S, Kim C, Wolmark N: Her2 status and benefit from adjuvant trastuzumab in breast cancer. N Engl J Med 2008;358:1409-1411.

-7 Luque-Cabal M, Garcia-Teijido P, Fernandez-Perez Y, Sanchez-Lorenzo L, Palacio-Vazquez I: Mechanisms behind the resistance to trastuzumab in her2-amplified breast cancer and strategies to overcome it. Clin Med Insights Oncol 2016;10:21-30.

8 Nahta R, Esteva FJ: Her2 therapy: Molecular mechanisms of trastuzumab resistance. Breast Cancer Res 2006;8:215.

-9 Lavaud P, Andre F: Strategies to overcome trastuzumab resistance in her2-overexpressing breast cancers: Focus on new data from clinical trials. BMC Med 2014;12:132.

10 Wong H, Leung R, Kwong A, Chiu J, Liang R, Swanton C, Yau T: Integrating molecular mechanisms and clinical evidence in the management of trastuzumab resistant or refractory her-2(+) metastatic breast cancer. Oncologist 2011;16:1535-1546.

-11 Blackwell KL, Burstein HJ, Storniolo AM, Rugo H, Sledge G, Koehler M, Ellis C, Casey M, Vukelja S, Bischoff J, Baselga J, O'Shaughnessy J: Randomized study of lapatinib alone or in combination with trastuzumab in women with erbb2-positive, trastuzumab-refractory metastatic breast cancer. J Clin Oncol 2010;28:11241130.

12 Geyer C E, Forster J, Lindquist D, Chan S, Romieu CG, Pienkowski T, Jagiello-Gruszfeld A, Crown J, Chan A, Kaufman B, Skarlos D, Campone M, Davidson N, Berger M, Oliva C, Rubin SD, Stein S, Cameron D: Lapatinib plus capecitabine for her2-positive advanced breast cancer. N Engl J Med 2006;355:2733-2743.

$\checkmark 13$ Verma S, Miles D, Gianni L, Krop I E, Welslau M, Baselga J, Pegram M, Oh DY, Dieras V, Guardino E, Fang L, Lu MW, Olsen S, Blackwell K, Group E S: Trastuzumab emtansine for her2-positive advanced breast cancer. N Engl J Med 2012;367:1783-1791.

14 Baselga J, Gelmon KA, Verma S, Wardley A, Conte P, Miles D, Bianchi G, Cortes J, McNally VA, Ross G, Fumoleau P, Gianni L: Phase ii trial of pertuzumab and trastuzumab in patients with human epidermal growth factor receptor 2-positive metastatic breast cancer that progressed during prior trastuzumab therapy. J Clin Oncol 2010;28:1138-1144. 


\section{Cellular Physiology Cell Physiol Biochem 2018;49:419-431 \begin{tabular}{l|l|l|l|}
\hline DOI: 10.1159/000492977 & 2018 The Author(s). Published by S. Karger AG, Basel \\
and Biochemistry.karger.com/cpb
\end{tabular} and Biochemistry

Sun et al.: tRNA-Derived Fragments as Novel Predictive Biomarkers for TrastuzumabResistant Breast Cancer

15 Goodarzi H, Nguyen HC, Zhang S, Dill BD, Molina H, Tavazoie S : Modulated expression of specific tRNAs drives gene expression and cancer progression. Cell 2016;165:1416-1427.

16 Mahlab S, Tuller T, Linial M: Conservation of the relative trna composition in healthy and cancerous tissues. RNA 2012;18:640-652.

17 Clarke CJ, Berg TJ, Birch J, Ennis D, Mitchell L, Cloix C, Campbell A, Sumpton D, Nixon C, Campbell K, Bridgeman VL, Vermeulen PB, Foo S, Kostaras E, Jones JL, Haywood L, Pulleine E, Yin H, Strathdee D, Sansom 0, et al.: The initiator methionine trna drives secretion of type ii collagen from stromal fibroblasts to promote tumor growth and angiogenesis. Curr Biol 2016;26:755-765.

18 Gebetsberger J, Polacek N: Slicing trnas to boost functional ncrna diversity. RNA Biol 2013;10:1798-1806. Thompson DM, Parker R: Stressing out over tRNA cleavage. Cell 2009;138:215-219.

-20 Lee Y, Shibata Y, Malhotra A, Dutta A: A novel class of small RNAs: tRNA-derived RNA fragments (trfs). Genes Dev 2009;23:2639-2649.

-21 Telonis AG, Loher P, Honda S, Jing Y, Palazzo J, Kirino Y, Rigoutsos I: Dissecting trna-derived fragment complexities using personalized transcriptomes reveals novel fragment classes and unexpected dependencies. Oncotarget 2015;6:24797-24822.

22 Zheng LL, Xu WL, Liu S, Sun WJ, Li JH, Wu J, Yang JH, Qu LH: Trf2cancer: A web server to detect tRNA-derived small rna fragments (trfs) and their expression in multiple cancers. Nucleic Acids Res 2016;44:gkw414.

23 Goodarzi H, Liu X, Nguyen HC, Zhang S, Fish L, Tavazoie SF: Endogenous tRNA-derived fragments suppress breast cancer progression via ybx1 displacement. Cell 2015;161:790-802.

-24 Green D, Fraser WD, Dalmay T: Transfer RNA-derived small rnas in the cancer transcriptome. Pflugers Arch 2016;468:1041-1047.

25 Anderson P, Ivanov P: tRNA fragments in human health and disease. FEBS Lett 2014;588:4297-4304.

-26 Sobala A, Hutvagner G: Small rnas derived from the 5' end of tRNA can inhibit protein translation in human cells. RNA Biol 2013;10:553-563.

-27 Kawaji H, Nakamura M, Takahashi Y, Sandelin A, Katayama S, Fukuda S, Daub CO, Kai C, Kawai J, Yasuda J, Carninci P, Hayashizaki Y: Hidden layers of human small RNAs. BMC Genomics 2008;9:157.

28 Li Z, Ender C, Meister G, Moore PS, Chang Y, John B: Extensive terminal and asymmetric processing of small rnas from rrnas, snornas, snrnas, and tRNAs. Nucleic Acids Res 2012;40:6787-6799.

29 Sun C, Fu Z, Wang S, Li J, Li Y, Zhang Y, Yang F, Chu J, Wu H, Huang X, Li W, Yin Y: Roles of tRNA-derived fragments in human cancers. Cancer Lett 2017;414:16-25.

-30 Saikia M, Jobava R, Parisien M, Putnam A, Krokowski D, Gao XH, Guan B J, Yuan Y, Jankowsky E, Feng Z: Angiogenin-cleaved tRNA halves interact with cytochrome c, protecting cells from apoptosis during osmotic stress. Mol Cell Biol2014;34:2450-2463.

-31 Olvedy M, Scaravilli M, Hoogstrate Y, Visakorpi T, Jenster G, Martens-Uzunova ES: A comprehensive repertoire of tRNA-derived fragments in prostate cancer. Oncotarget 2016;7:24766-24777.

-32 Nagy P, Friedlander E, Tanner M, Kapanen AI, Carraway K L, Isola J, Jovin TM: Decreased accessibility and lack of activation of erbb2 in jimt-1, a herceptin-resistant, muc4-expressing breast cancer cell line. Cancer Res 2005;65:473-482.

-33 Nam S, Chang HR, Jung HR, Gim Y, Kim NY, Grailhe R, Seo HR, Park HS, Balch C, Lee J, Park I, Jung SY, Jeong KC, Powis G, Liang H, Lee ES, Ro J, Kim YH: A pathway-based approach for identifying biomarkers of tumor progression to trastuzumab-resistant breast cancer. Cancer Lett 2015;356:880-890.

34 Zhu A, Li Y, Song W, Xu Y, Yang F, Zhang W, Yin Y, Guan X: Antiproliferative effect of androgen receptor inhibition in mesenchymal stem-like triple-negative breast cancer. Cell Physiol Biochem 2016;38:10031014.

-35 Qian Y, Shi D, Qiu J, Zhu F, Qian J, He S, Shu Y, Yin Y, Chen X: Obrb downregulation increases breast cancer cell sensitivity to tamoxifen. Tumour Biol 2015;36:6813-6821.

-36 Loher P, Telonis A G, Rigoutsos I: Mintmap: Fast and exhaustive profiling of nuclear and mitochondrial trna fragments from short rna-seq data. Sci Rep 2017;7:41184.

-37 Pliatsika V, Loher P, Magee R, Telonis AG, Londin E, Shigematsu M, Kirino Y, Rigoutsos I: Mintbase v2.0: A comprehensive database for trna-derived fragments that includes nuclear and mitochondrial fragments from all the cancer genome atlas projects. Nucleic Acids Res 2018;46:D152-D159.

-38 Pliatsika V, Loher P, Telonis AG, Rigoutsos I: Mintbase: A framework for the interactive exploration of mitochondrial and nuclear trna fragments. Bioinformatics 2016;32:2481-2489. 


\section{Cellular Physiology Cell Physiol Biochem 2018;49:419-431 \begin{tabular}{c|c|c|} 
DOI: 10.1159/000492977 & O 2018 The Author(s). Published by S. Karger AG, Basel \\
mwwhargercom/cpb
\end{tabular} and Biochemistry}

Sun et al.: tRNA-Derived Fragments as Novel Predictive Biomarkers for TrastuzumabResistant Breast Cancer

-39 Zhang MY, Li SH, Huang GL, Lin GH, Shuang Z, Lao XM, Xu L, Lin XJ, Wang HY, Li SP: Identification of a novel microrna signature associated with intrahepatic cholangiocarcinoma (icc) patient prognosis. BMC Cancer 2015;15:64.

-40 Honda S, Loher P, Shigematsu M, Palazzo J P, Suzuki R, Imoto I, Rigoutsos I, Kirino Y: Sex hormonedependent tRNA halves enhance cell proliferation in breast and prostate cancers. Proc Natl Acad Sci U S A 2015;112:E3816-3825.

41 Lv M, Xu P, Wu Y, Huang L, Li W, Lv S, Wu X, Zeng X, Shen R, Jia X, Yin Y, Gu Y, Yuan H, Xie H, Fu Z: Lncrnas as new biomarkers to differentiate triple negative breast cancer from non-triple negative breast cancer. Oncotarget 2016;7:13047-13059.

42 Keam SP, Hutvagner G: tRNA-derived fragments (trfs): Emerging new roles for an ancient RNA in the regulation of gene expression. Life (Basel) 2015;5:1638-1651.

43 Zhao H, Bojanowski K, Ingber DE, Panigrahy D, Pepper MS, Montesano R, Shing Y: New role for tRNA and its fragment purified from human urinary bladder carcinoma conditioned medium: Inhibition of endothelial cell growth. J Cell Biochem 1999;76:109-117.

-44 Martens-Uzunova ES, Jalava SE, Dits NF, van Leenders GJ, Moller S, Trapman J, Bangma CH, Litman T, Visakorpi T, Jenster G: Diagnostic and prognostic signatures from the small non-coding RNA transcriptome in prostate cancer. Oncogene 2012;31:978-991.

-45 Speer J, Gehrke CW, Kuo KC, Waalkes TP, Borek E: Trna breakdown products as markers for cancer. Cancer 1979;44:2120-2123.

46 Borek E, Baliga BS, Gehrke CW, Kuo CW, Belman S, Troll W, Waalkes TP: High turnover rate of transfer RNA in tumor tissue. Cancer Res 1977;37:3362-3366.

-47 Dhahbi JM, Spindler SR, Atamna H, Boffelli D, Martin DI: Deep sequencing of serum small RNAs identifies patterns of 5' tRNA half and yrna fragment expression associated with breast cancer. Biomark Cancer 2014;6:37-47.

-48 Nientiedt M, Deng M, Schmidt D, Perner S, Muller S, Ellinger J: Identification of aberrant tRNA-halves expression patterns in clear cell renal cell carcinoma. Sci Rep 2016;6:37158.

49 Witwer KW, Buzas EI, Bemis LT, Bora A, Lasser C, Lotvall J, Nolte-'t Hoen EN, Piper MG, Sivaraman S, Skog J, Thery C, Wauben MH, Hochberg F: Standardization of sample collection, isolation and analysis methods in extracellular vesicle research. J Extracell Vesicles 2013; DOI:10.3402/jev.v210.20360.

-50 Jia QW, Chen ZH, Ding XQ Liu JY, Ge PC, An FH, Li LH, Wang LS, Ma WZ, Yang ZJ, Jia EZ: Predictive effects of circulating miR-221, miR-130a and miR-155 for coronary heart disease: A multi-ethnic study in china. Cell Physiol Biochem 2017;42:808-823.

51 Xie T, Huang M, Wang Y, Wang L, Chen C, Chu X: Micrornas as regulators, biomarkers and therapeutic targets in the drug resistance of colorectal cancer. Cell Physiol Biochem 2016;40:62-76.

52 Ivanov P, Emara MM, Villen J, Gygi S , Anderson P: Angiogenin-induced trna fragments inhibit translation initiation. Mol Cell 2011;43:613-623.

53 Yamasaki S, Ivanov P, Hu GF, Anderson P: Angiogenin cleaves tRNA and promotes stress-induced translational repression. J Cell Biol 2009;185:35-42.

-54 Haussecker D, Huang Y, Lau A, Parameswaran P, Fire AZ, Kay MA: Human tRNA-derived small RNAs in the global regulation of rna silencing. RNA 2010;16:673-695.

55 Yeung ML, Bennasser Y, Watashi K, Le S, Houzet L, Jeang KT: Pyrosequencing of small non-coding RNAs in hiv-1 infected cells: Evidence for the processing of a viral-cellular double-stranded rna hybrid. Nucleic Acids Res 2009;37:6575-6586.

56 Balatti V, Nigita G, Veneziano D, Drusco A, Stein GS, Messier TL, Farina NH, Lian JB, Tomasello L, Liu CG, Palamarchuk A, Hart JR, Bell C, Carosi M, Pescarmona E, Perracchio L, Diodoro M, Russo A, Antenucci A, Visca P, et al.: TsRNA signatures in cancer. Proc Natl Acad Sci U S A 2017;114:8071-8076.

57 Dhahbi JM, Spindler SR, Atamna H, Yamakawa A, Boffelli D, Mote P, Martin DI: 5' tRNA halves are present as abundant complexes in serum, concentrated in blood cells, and modulated by aging and calorie restriction. BMC Genomics 2013;14:298. 